\title{
O significado atribuído a 'ser idoso' por trabalhadores de instituições de longa permanência*
}

\author{
THE MEANING ATTRIBUTED TO ‘BEING OLD'BY WORKERS OF \\ LONG TERM CARE NURSING INSTITUTIONS
}

\author{
EL SIGNIFICADO ATRIBUÍDO A 'SER ANCIANO' POR TRABAJADORES \\ DE INSTITUCIONES DE LARGA PERMANENCIA
}

Priscilleyne Ouverney Reis ${ }^{1}$, Maria Filomena Ceolim²

\section{RESUMO}

Trata-se de um estudo do tipo exploratório e descritivo, com abordagem quantitativa, cujo objetivo foi identificar o significado atribuído a 'ser idoso' por 50 trabalhadores que prestam cuidados diretos de enfermagem a idosos institucionalizados. Os dados foram coletados com a versão da 'Escala Diferencial Semântica' validada no Brasil. A categoria ocupacional predominante entre os sujeitos foi a de 'auxiliar de enfermagem', seguida pela de 'serviços gerais'. A maioria dos sujeitos atribuiu ao idoso os adjetivos: bonito, carinhoso, doce, sábio, interessante, com senso de oportunidade, humilde e conformado. Os atributos destrutivos: inseguro, fraco, crítico e intrometido também foram citados. Verificou-se que os sujeitos selecionaram atributos que, embora positivos, são relacionados à docilidade e passividade.

\section{DESCRITORES}

Idoso.

Envelhecimento.

Asilos para idosos.

Formação de conceito.

Pessoal da saúde.

\section{ABSTRACT}

This is a descriptive and explorative study with a quantitative approach aimed at identifying the meaning of 'being old' according to 50 health-care workers responsible for direct nursing care to elderly residents in nursing homes. Data was obtained by means of the Portuguese version of the 'Semantic Differential Scale' validated in Brazil. Subjects were predominantly nursing assistants and 'general services' workers. The majority of them conferred to the elderly the adjectives beautiful, tender, sweet, wise, interesting, with sense of opportunity, humble and resigned. The attributes destructive, insecure, critic and intrusive were also cited. Most subjects selected adjectives that, in spite of being positive, are also related with docility and passivity.

\section{KEY WORDS}

Aged.

Aging.

Homes for the aged.

Concept formation.

Health personnel

\section{RESUMEN}

Se trata de un estudio de tipo descriptivo - exploratorio, con abordaje cuantitativo, cuyo objetivo fue identificar el significado atribuido a 'ser anciano' por 50 trabajadores que prestan cuidados directos de enfermería a ancianos institucionalizados. Los datos fueron recolectados con la versión de la 'Escala Diferencial Semántica' validada en el Brasil. La categoría ocupacional predominante entre los sujetos fue la de 'auxiliar de enfermería', seguida por la de 'servicios generales'. La mayoría de los sujetos atribuyó al anciano los adjetivos: bonito, cariñoso, dulce, sabio, interesante, con sentido de oportunidad, humilde y conformista. Los atributos destructivo, inseguro, frágil, crítico y entrometido también fueron citados. Se verificó que los sujetos seleccionaron atributos que, aunque positivos, están relacionados a docilidad y pasividad.

\section{DESCRIPTORES}

Anciano.

Envejecimiento.

Hogares para ancianos.

Formación de concepto.

Personal de salud.
* Extraído do estudo intitulado "A atenção à saúde do idoso em instituições totais de um município do

interior do estado de São Paulo: perfil dos prestadores de

cuidados" desenvolvido com bolsa de Iniciação Científica financiada pela FAPESP.

1 Enfermeira do Centro de Atenção Integral à Saúde da Mulher da Universidade Estadual de Campinas (UNICAMP). Mestre em Enfermagem pelo Programa de Pós-graduação em Enfermagem da

Faculdade de

Ciências Médicas

(FCM) - UNICAMP. ouverneyreis@ yahoo.com.br

2 Professora Doutora do Departamento de Enfermagem da FCM/UNICAMP.

Enfermeira.

fceolim@

fcm.unicamp.br 


\section{INTRODUÇÃO}

É notória a persistência de crenças e estereótipos sobre o significado de 'ser velho' e sobre o comportamento do idoso. Ainda hoje, a prestação de cuidados sofre a influência nociva da crença, cristalizada desde a Antigüidade, de que o envelhecimento é um processo degenerativo, oposto a qualquer progresso ou desenvolvimento ${ }^{(1)}$, resultando no rótulo do idoso como um 'adulto menos capaz'. O desconhecimento de aspectos fisiológicos, psicológicos, emocionais e sociais específicos do idoso, por parte dos profissionais que prestam assistência, contribui para as deficiências na compreensão do idoso e para o atendimento inadequado. $\mathrm{O}$ potencial para o autocuidado e a independência não será estimulado, ou será anulado pelo profissional despreparado que assiste o idoso.

A dependência está presente desde o nascimento, não sendo um aspecto específico da terceira idade. A diferença é que, na infância, a dependência caminha para a competência, enquanto que na velhice ocorre o inverso, ampliando-se e tendendo a ser definitiva ou permanente ${ }^{(1)}$. Atualmente, considera-se muitas vezes que ser velho significa ser excluído, principalmente do universo do trabalho, classificando-se deste modo o idoso como improdutivo. A mídia explora temas relacionados à terceira idade geralmente a partir de uma visão sensacionalista, prejudicando a imagem do idoso por ressaltar somente os aspectos que atraem o público. Nos últimos 50 anos do século $\mathrm{XX}$ as pesquisas científicas em Gerontologia, tanto norte-americanas quanto européias, foram dotadas de grande preconceito $\mathrm{e}$ influenciaram as pesquisas realizadas no Brasil. Somente na década de 80 houve um maior interesse quanto ao processo de envelhecimento enquanto etapa natural do desenvolvimento do ser humano, ganhando também o domínio público ${ }^{(2-5)}$.

No Brasil, a escassez de programas sociais e de saúde voltados tanto para a promoção da independência como para a manutenção do idoso dependente no seu domicílio levam, em muitos casos, à internação precoce em instituições de longa permanência como casas de repouso e asilos, que deveriam ser utilizadas como última alternativa por anciãos muito frágeis e dependentes que não pudessem ser mantidos em seus lares ${ }^{(6-7)}$. Estes estabelecimentos são a modalidade mais antiga de atenção ao idoso fora da família, mas têm o inconveniente de levar esta população ao isolamento e à inatividade física em decorrência do manejo técnico inadequado e dos custos altos dos serviços de apoio( ${ }^{(6}$. É importante assinalar que apesar da Secretaria do Estado de Assistência Social ter definido as normas de funcionamento de serviços de atenção ao idoso, através da Portaria ${ }^{\circ} 73$ de 10 de maio de $2001^{(8)}$, a proliferação destes serviços vem ocorrendo, muitas vezes, sem cumprir requisitos mínimos ${ }^{(6-9)}$.
Nos últimos anos, diversos debates tem sido realizados com intuito de introduzir novos conceitos no campo da Gerontologia. Um destes trata a respeito da nomenclatura das instituições para idosos. Neste trabalho, a expressão Instituição de Longa Permanência (ILP) foi utilizada para designar os locais físicos equipados para atender idosos em regime de internato, mediante pagamento ou não, durante um período determinado ou não ${ }^{(7)}$.

Considerando as peculiaridades e demandas dos idosos, o enfermeiro pode e deve atuar em diversas vertentes, tanto contribuindo para minimizar as condições que levam à institucionalização desnecessária, assegurando a participação do idoso e da família na escolha da modalidade, quanto buscando estratégias voltadas para a melhoria dos cuidados de enfermagem oferecidos nas instituições, de forma a respeitar a história de vida, os valores e os hábitos culturais das pessoas envolvidas e, portanto, melhorando a própria qualidade de vida dos seus clientes ${ }^{(10-12)}$.

Desta forma, visando contribuir com estratégias que promovam, a médio e longo prazo, a melhoria da qualidade dos cuidados de enfermagem oferecidos aos idosos, este trabalho teve como objetivo identificar o significado atribuído a 'ser idoso' por trabalhadores que prestam cuidados diretos de enfermagem a idosos em instituições de longa permanência.

\section{METODOLOGIA}

A pesquisa foi realizada nos anos de 2000 e 2001, em cinco instituições totais que atendiam idosos, cadastradas na prefeitura do município de Campinas, estado de São Pau$1 \mathrm{o}^{(10)}$. Trata-se de um estudo exploratório e descritivo, com abordagem quantitativa.

Os sujeitos da pesquisa foram os funcionários das cinco instituições que atendiam aos seguintes critérios de inclusão: realizar cuidados diretos de enfermagem de qualquer natureza ou complexidade com os idosos institucionalizados, podendo pertencer a uma das diversas categorias de enfermagem, atualmente legais ou não (enfermeiros, técnicos, auxiliares ou atendentes de enfermagem), ou a outra categoria profissional ou ocupacional, ou, ainda, serem leigos; e participar voluntariamente, após assinatura de Termo de Consentimento Livre e Esclarecido.

Constituíram critérios de exclusão: recusar-se a participar do estudo; encontrar-se em período de férias ou de licença de qualquer natureza ou não devolver o questionário respondido após três solicitações.

Os dados foram coletados por meio de um instrumento preenchido pelos próprios sujeitos, composto por três partes distintas: a Parte I, adaptada de outro autor ${ }^{(13)}$, permitiu 
caracterizar os sujeitos quanto a aspectos demográficos e às suas atividades na instituição; a Parte II, modificada de outro trabalho ${ }^{(14)}$, foi utilizada para identificar as dificuldades enfrentadas pelos sujeitos no cuidado ao idoso, bem como suas necessidades de conhecimento específico sobre o envelhecimento; a Parte III consistiu da aplicação da Escala Diferencial Semântica ${ }^{(15-16)}$.

Na Escala Diferencial Semântica são apresentados 40 pares de adjetivos bipolares, ou seja, com conotação positiva e negativa, dispostos por sorteio, ao acaso. Os adjetivos correspondem a atributos que visam caracterizar os idosos em três grandes aspectos: capacidade de perseguir objetivos, resolver problemas e adaptar-se a mudanças; desejabilidade; aceitação social. O sujeito escolhe a polaridade para cada par de adjetivos apresentados e atribui, por meio de uma escala numérica, grau de maior ou menor intensidade à escolha.

A Escala é dividida em três grandes categorias e cinco subcategorias, segundo a proposta do autor que a validou ${ }^{(15)}$. Cada categoria engloba um número diferente de pares de atributos correspondendo a determinadas características do idoso. A primeira é Velho Desejável/Velho Indesejável, que inclui as sub-categorias Geratividade/Estagnação, Autonomia/Dependência e Integração/Isolamento. A segunda categoria é Velho Adaptado/Velho Desadaptado, com as subcategorias Valorização/Desvalorização e Aceitação/Rejeição. A terceira categoria é Velho que não incomoda/Velho a ser evitado.

Para a coleta de dados, os sujeitos que atendiam aos critérios de inclusão na pesquisa receberam os instrumentos com orientações para o preenchimento. Marcou-se uma data para a devolução dos instrumentos sendo, em média, dez dias após a entrega. Foram entregues 66 instrumentos e devolvidos 50 (76\%).

O tratamento estatístico empregue foi, predominantemente, a análise descritiva. Devido ao número de sujeitos estudados, os testes estatísticos não paramétricos de Kruskal-Wallis e Mann-Whitney foram utilizados para identificar possível associação entre os resultados obtidos com a Escala Diferencial Semântica e: as características dos sujeitos; o grau de dificuldade atribuído pelos sujeitos a diversas atividades de cuidado que ofereciam ao idoso. Foram consideradas significativas as diferenças entre os grupos quando o valor de 'p' (nível crítico) obtido com o teste aplicado foi menor ou igual a 0,05 .

Os resultados da Escala Diferencial Semântica foram analisados atribuindo-se pontuação de um (1) a sete (7) a cada par de adjetivos bipolares correspondentes aos atributos usados para caracterizar o 'idoso'. Foi realizado tratamento estatístico para que os adjetivos mais positivos correspondessem sempre à pontuação mais alta. Para isto, quando o adjetivo de conotação negativa se encontrava no polo de maior pontuação, o escore marcado pelo sujeito foi submetido à fórmula $\mathrm{X}=8$ - $\mathrm{Y}$, em que $\mathrm{Y}$ é o escore marcado e $\mathrm{X}$ corresponde ao escore resultante ${ }^{(16)}$.

Esta pesquisa foi aprovada pelo Comitê de Ética em Pesquisa da Faculdade de Ciências Médicas da Universidade Estadual de Campinas (processo $\mathrm{n}^{\circ} 160 / 2000$ ), encontrandose de acordo com as disposições da Resolução 196/96 do Conselho Nacional de Saúde. O contato inicial com as instituições foi feito por meio de carta enviada aos dirigentes, que deram seu consentimento formal para o estudo. Os funcionários assinaram Termo de Consentimento Livre e Esclarecido, no qual foi explicitada a finalidade da pesquisa e assegurado o anonimato dos participantes e da instituição, bem como o caráter voluntário da participação.

\section{RESULTADOS}

Participaram 50 sujeitos pertencentes, predominantemente, ao sexo feminino (94\%), com idade variando entre 21 e 63 anos. A maior parte encontrava-se na faixa etária entre 31 e 40 anos (36\%), seguida pelas faixas de 21 a 30 anos e de 41 a 50 anos, com $23 \%$ dos sujeitos em cada uma. Quase a metade dos sujeitos (42\%) referiu não haver completado o antigo primeiro grau, enquanto $27 \%$ possuíam oito anos de escolaridade, correspondendo ao antigo primeiro grau completo, e nenhum sujeito referiu o antigo terceiro grau, completo ou incompleto.

Segundo o relato dos sujeitos, a categoria predominante eram os auxiliares de enfermagem (40\%), seguidos por aqueles que se inseriam, de acordo com eles mesmos, nas seguintes categorias: serviços gerais $(32 \%)$; atendentes de enfermagem (10\%); técnicos de enfermagem (4\%), cozinheiras $(4 \%)$ e assistente administrativo $(2 \%)$. As diferentes categorias estavam distribuídas de forma desigual entre as cinco instituições.

Uma grande proporção de sujeitos (90\%) relatou nunca ter feito um curso específico que os capacitasse a cuidar de idosos.

Quanto ao tempo de experiência na assistência aos idosos, $40 \%$ dos sujeitos referiam desempenhar este tipo de atividade há cinco anos ou mais, seguindo-se o grupo que trabalhava há um período compreendido entre 13 meses e cinco anos (32\%). Quando questionados sobre os motivos pelos quais trabalhavam na assistência aos idosos, o desemprego (36\%) e a relação afetuosa com os idosos (35\%) foram os mais citados.

A maior parte dos sujeitos referiu nenhuma, pouca ou pouquíssima dificuldade na maior parte das atividades realizadas no cuidado com o idoso. A atividade referente ao lidar com a morte foi a única em que um maior número de sujeitos $(30 \%)$ referiu muita e muitíssima dificuldade. 
Os 40 atributos da Escala Diferencial Semântica, utilizados para que o sujeito descrevesse o idoso segundo sua percepção, foram agrupados nas categorias e subcategorias apresentadas anteriormente ${ }^{(14)}$. Os escores mais elevados representam as avaliações mais positivas.
Destacam-se alguns dos atributos que, pela porcentagem de respostas com elevado grau de polaridade (positiva ou negativa), permitiram caracterizar a percepção dos sujeitos a respeito do idoso. Esta porcentagem é apresentada na Tabela 1, segundo a polaridade das respostas, destacandose apenas as mais negativas (escores 1 e 2) e as mais positivas (escores 6 e 7).

Tabela 1 - Distribuição percentual das respostas segundo polaridade atribuída na Escala diferencial semântica em alguns dos atributos imputados ao idoso - Campinas - 2000

\begin{tabular}{lcc}
\hline \multirow{2}{*}{ Categorias } & Polaridade positiva & Polaridade negativa \\
\cline { 2 - 3 } & Respostas (\%) & Respostas (\%) \\
\hline Construtivo/Destrutivo & 7,1 & 57,1 \\
Seguro/Inseguro & 20,5 & 52,2 \\
Forte/Fraco & 15,9 & 40,9 \\
Condescendente/Crítico & 9,7 & 36,6 \\
Discreto/Intrometido & 16,7 & 52,4 \\
Bonito/Feio & 49,0 & 22,7 \\
Carinhoso/Frio & 41,5 & 24,4 \\
Doce/Amargo & 60,8 & 2,3 \\
Sábio/Ignorante & 50,0 & 9,0 \\
Interessante/Desinteressante & 52,5 & 4,7 \\
Com senso de oportunidade/Chato & 34,1 & 6,8 \\
Humilde/Arrogante & 38,1 & 19,1 \\
Conformado/Queixoso & 42,2 & 22,7 \\
\hline
\end{tabular}

Analisando-se a Tabela 1, verifica-se que os sujeitos, em sua maioria, classificaram o idoso como: bonito, carinhoso, doce, sábio, interessante, com senso de oportunidade, humilde e conformado. Entretanto, também foi grande a porcentagem dos que atribuíram ao idoso características como destrutivo, inseguro, fraco, crítico e intrometido.
O Quadro 1 contém as médias, desvios-padrão e medianas dos valores imputados pelos sujeitos a cada uma das três categorias definidas de atributos, bem como às subcategorias.

Quadro 1 - Escores imputados pelos sujeitos $(n=50)$ aos atributos da Escala diferencial semântica: média, desviopadrão e mediana - Campinas - 2000

\begin{tabular}{|c|c|c|c|}
\hline \multirow{2}{*}{ Categorias e Subcategorias de Atributos da Escala Diferencial Semântica } & \multicolumn{3}{|c|}{ Escores } \\
\hline & Média & D.P. & Mediana \\
\hline $\begin{array}{l}\text { Velho Desejável/ Velho Indesejável* (escore máximo 175) } \\
\text { Geratividade/Estagnação** (escore máximo: 77) } \\
\text { Autonomia/Dependência** (escore máximo: 35) } \\
\text { Integração/Isolamento** (escore máximo: 63) }\end{array}$ & $\begin{array}{r}103,0 \\
44,5 \\
19,0 \\
39,5\end{array}$ & $\begin{array}{l}22,0 \\
10,0 \\
6,4 \\
10,1\end{array}$ & $\begin{array}{l}98,0 \\
44,5 \\
19,0 \\
36,0\end{array}$ \\
\hline $\begin{array}{l}\text { Velho Adaptado/ Velho Desadaptado* (escore máximo 56) } \\
\text { Valorização/Desvalorização** (escore máximo: 28) } \\
\text { Aceitação/Rejeição** (escore máximo: 28) }\end{array}$ & $\begin{array}{l}34,4 \\
17,6 \\
16,8\end{array}$ & $\begin{array}{l}7,7 \\
4,6 \\
4,1\end{array}$ & $\begin{array}{l}32,5 \\
16,0 \\
16,0\end{array}$ \\
\hline Velho que não incomoda/Velho a ser evitado* (escore máximo 49) & 28,5 & 6,3 & 29,5 \\
\hline
\end{tabular}

*: categoria de atributos $\quad * *$ : subcategorias englobadas pela categoria acima

D.P.: Desvio-padrão

Verificou-se que a média dos escores encontra-se acima da metade do valor máximo possível, portanto acima do valor neutro, revelando a tendência dos sujeitos a classificar o idoso escolhendo os atributos mais positivos.

Identificou-se associação estatisticamente significativa (p-valor < 0,04 ao teste de Kruskal-Wallis) entre a faixa etária dos sujeitos e o significado atribuído a 'ser idoso': sujeitos pertencentes à faixa etária entre 21 e 30 anos consideravam o idoso como desejável, mais do que aqueles das faixas entre 51 e 60 anos, e entre 61 e 70 anos.

Observou-se também associação estatisticamente significativa ( $\mathrm{p}<0,05$ segundo os testes de Kruskal-Wallis e de Mann-Whitney) entre o grau de dificuldade relatado pelos sujeitos para diversas atividades do cuidado e a pontuação

$\begin{aligned} \text { Rev Esc Enferm USP } & \text { O significado atribuído a 'ser idoso' por trabalhadores } \\ 2007 ; 41(1): 57-64 . & \text { de instituições de longa permanência } \\ \text { Rww.ee.usp.br/reeusp/ } & \text { Reis PO, Ceolim MF. }\end{aligned}$


obtida na Escala, nas seguintes situações: sujeitos que relataram graus mais elevados de dificuldade nas atividades de locomoção, comunicação, higiene corporal, eliminação urinária e eliminação fecal caracterizaram o idoso mais como indesejável do que como desejável; sujeitos que relataram graus mais elevados de dificuldade com atividades de mobilização, estado mental e higiene oral caracterizaram o idoso mais como desadaptado do que como adaptado; sujeitos que relataram graus mais elevados de dificuldade com atividade de higiene corporal caracterizaram o idoso mais como o velho a ser evitado do que como aquele que não incomoda.

Deve-se destacar, entretanto, que somente pequeno número de sujeitos apontou grau elevado de dificuldade em qualquer atividade de cuidado, o que indica que é necessário cautela ao avaliar estes achados, apesar da significância estatística.

Dentre os estudados, 54\% (27/50) referiam algum tipo de experiência anterior com idosos, na sua vida familiar ou pessoal, sendo que $85 \%$ desses (23/27) apresentaram respostas que caracterizavam o relacionamento como positivo.

\section{DISCUSSÃO}

As características demográficas e sociais observadas na população de trabalhadores estudados apresentam-se semelhantes às obtidas em outros estudos relativos ao cuidado de enfermagem oferecido ao idoso institucionalizado.

O predomínio dos trabalhadores do sexo feminino é um achado que vem de encontro à literatura sobre a crescente inserção da mulher no mercado de trabalho a partir da segunda metade do século XX, cujas principais causas seriam: a deterioração dos salários reais impelindo a mulher a participar do orçamento da família; e a crescente participação das mulheres em espaços públicos, especialmente na enfermagem, a qual é historicamente uma profissão feminina ${ }^{(17)}$.

Quanto à qualificação dos trabalhadores que prestavam os cuidados de enfermagem, também os resultados se mostraram de acordo com a literatura a respeito. Os estudos existentes no Brasil têm mostrado que a situação atual é de praticamente total falta de qualificação para o atendimento adequado ao idoso, seja em âmbito domiciliar, hospitalar ou institucional ${ }^{(9-10,13-14,18-20)}$. É importante assinalar que a formação acadêmica dos enfermeiros, ainda hoje, não contempla as necessidades impostas pela prática profissional e ainda está ancorada em estereótipos e preconceitos negativos ${ }^{(21-23)}$.

A prática de enfermagem nas instituições tem sido realizada por atendentes de enfermagem que, segundo a Lei do Exercício Profissional de Enfermagem (Lei no 7.498/86, regulamentada pelo Decreto n $4406 / 871)^{(24)}$, não podem mais exercer atividades de enfermagem, mas que são muitas vezes contratados formalmente para prestar 'serviços gerais'.
Verificou-se em outro estudo ${ }^{(17)}$ que tal achado se agravava ainda mais pela ausência de enfermeiros nos cargos de chefia, o que está de acordo com esta pesquisa. Concluiu ainda que a força de trabalho era não qualificada, e os serviços prestados, em geral, eram insuficientes e inadequados ${ }^{(18)}$.

Esses aspectos reforçam a necessidade do profissional enfermeiro no contexto das instituições, não apenas no cuidado direto como também no gerenciamento da assistência de enfermagem e nas tarefas de educação em serviço. Entretanto, não foi este o panorama revelado neste estudo, que sugere uma situação deficitária em termos de quantidade e possivelmente de qualidade no atendimento ao idoso nas instituições estudadas.

O significado atribuído pelos funcionários a 'ser idoso', na Escala Diferencial Semântica, vem confirmar a manutenção de diversos estereótipos entre estes profissionais. Verificou-se que se destacam os atributos doce, carinhoso, conformado e humilde, caracterizando a velhice dócil e submissa, dependente, tranqüila. Estudos realizados com enfermeiros em unidades básicas de saúde mostraram resultados semelhantes aos deste estudo. Os enfermeiros entrevistados tinham a imagem de que os idosos eram carentes, tolerantes, pacientes, compreensivos e gratos ${ }^{(23)}$. É interessante notar que a interpretação do 'ser idoso' foi mantida, apesar de referir-se a idosos não institucionalizados. Este achado reforça a necessidade de mudança na formação dos profissionais.

A análise mais detalhada das respostas dos sujeitos deste estudo sobre dificuldades que encontram ao prestar o cuidado contribui por sua vez no entendimento dos atributos que predominaram ao classificar o idoso. As respostas sugerem o que a literatura denomina por vitimização dos idosos, ou seja, o fato de vê-los como 'coitadinhos', contribuindo para que diminua ainda mais sua autonomia e independência ${ }^{(25)}$. Um dos entrevistados, ao ser questionado sobre o tipo de dificuldade encontrada no cuidado ao idoso, respondeu:

\section{É fácil se comunicar com o idoso devido o problema de que depois de uma certa idade eles viram criança novamente, aí fica mais fácil adquirir a confiança deles (D1)}

Outras citações que sugerem infantilização e vitimização também são referidas por outros funcionários nas diferentes instituições estudadas. Algumas são apresentadas a seguir:

...gosto de conversar com todos e fazer brincadeiras, procuro deixá-los mais alegres por uns instantes. Coitados, muitos deles tiveram uma vida difícil no passado, agora estão nas suas etapas finais, acho que eu ou qualquer outra pessoa devemos ter um pouco mais de paciência para que eles não se sintam mais tristes e inúteis (como eu já havia dito antes, eles viram crianças novamente é assim que todos nós também iremos ficar um dia)...(D1). 
... as pessoas se tornam idosas a partir do momento da rejeição familiar, falta de entrosamento social, sentindo-se solitário, inútil e incomprendido (D2)

... amo demais essas crianças grandes: os idosos (D3)

... cada um deles tem uma mania diferente.... as vezes chega a parecer que voltaram a ser crianças, e temos que respeitar e aceitar...(D4)

As pessoas quando envelhecem passam a depender de ajuda, é muito difícil para eles...(D5)

O fato de uma pessoa dizer que o velho não deve ser tratado como criança não o levará, necessariamente, a não agir dessa forma na prática ${ }^{(25)}$. Esta pessoa, muitas vezes, utiliza diminutivos, apelidos e expressões infantilizadoras ou familiaridade exagerada no seu relacionamento com o idoso. Esta atitude poderia estar associada à crença de que agir desta forma é sinal de afeto e compaixão, e que é dessa forma que os velhos devem e gostam de ser tratados ${ }^{(25)}$.

A sociedade atual valoriza essencialmente a juventude, mesmo sabendo que envelhecimento é um processo normal e que afeta todos os seres humanos e, tendo uma visão negativa deste período da vida, faz em parte com que os próprios velhos detenham esta imagem ${ }^{(26)}$, que constitui uma das idéias preconcebidas sobre a velhice. O desconhecimento deste processo leva a absorver e transmitir falsas percepções que contribuem, direta ou indiretamente, para isolar e acelerar o processo de envelhecimento. Os trabalhadores da área da saúde, como parte integrante desta sociedade, acabariam por absorver alguns destes 'pré-conceitos'.

Há estudos ${ }^{(27)}$ que identificaram sete mitos mais freqüentemente associados aos idosos. Estes mitos indicam o idoso como em sua maioria senis ou doentes; infelizes; não tão produtivos quanto os jovens; a maior parte doente e necessitando de ajuda para suas atividades cotidianas; mantêm obstinadamente seus hábitos de vida, são conservadores e incapazes de mudar; e finalmente que todos os idosos se assemelham e que todos estão isolados e sofrem de solidão. Entretanto, o mito da dependência, talvez um dos mais impregnados na sociedade, é posto em dúvida por trabalhos que apontam ${ }^{(27)}$ que $80 \%$ dos idosos são capazes de realizar as atividades de vida diária sem ajuda, uma vez que estes são suficientemente saudáveis e autônomos para tal.

A respeito dos mitos, o autor ${ }^{(27)}$ conclui com a seguinte afirmação:

...os mitos muitas vezes estão ligados ao desconhecimento do processo de envelhecer. Desta forma, a velhice está muitas vezes ligada à doença, ao aborrecimento, ao egoísmo, a impotência sexual, à rigidez de caráter, às rugas e aos cabelos brancos. Efetivamente só as rugas e os cabelos brancos é que são próprios das pessoas que enveIhecem. Todas as outras características se referem a todos os grupos etários, sem exceção. Apenas a nossa ignorância nos faz atribuí-los exclusivamente aos idosos.
Destaca-se ainda que o sistema social das instituições não privilegia nem incentiva o comportamento de independência e autonomia por parte dos idosos ${ }^{(25)}$. $\mathrm{O}$ incentivo à dependência é o que pode ser observado. Em um estudo onde se verificaram os padrões de interação entre duas atendentes não especializadas e 27 idosos, em uma instituição mantida por organização filantrópica ${ }^{(10)}$, os achados mostraram supremacia do padrão de manutenção de dependência, o que significa que, mesmo estando os idosos aptos a realizarem suas atividades de vida diária como banho, alimentação e medicação, mantinham o comportamento de dependência, estimulados pelos funcionários.

Autores relatam que o cuidador, ao supostamente agir no interesse dos idosos, muitas vezes na verdade os trata como crianças pequenas, não proporcionando a eles oportunidades de fazer escolhas e negando, portanto o direito à autonomia, atitude que pode ser considerada como uma forma de exercício de controle sobre o idoso ${ }^{(28)}$. Estes aspectos poderiam estar presentes, de forma velada, quando se classifica o idoso com atributos que se associam à docilidade, à submissão e ao conformismo, como nesta investigação.

Cabe ainda destacar a associação encontrada entre a faixa etária mais jovem e a escolha dos atributos que classificavam o idoso como desejável, presente com maior freqüência do que entre os funcionários mais idosos, achado que contradiz o resultado de outros estudos ${ }^{(16,29)}$. Em um deles, realizado no Brasil( ${ }^{(16)}$, o autor comparou os escores obtidos na Escala Diferencial Semântica por um grupo de idosos e um grupo de adultos jovens, estudantes de enfermagem, verificando que os primeiros obtiveram uma pontuação significativamente mais elevada em diversas subcategorias da Escala, ou seja, atribuíam ao ser 'idoso' um caráter mais positivo do que os estudantes de enfermagem. Em outro país, uma pesquisa realizada com alunos de nível médio no final da década de $90^{(29)}$, mostrou que os estudantes caracterizavam os idosos por possuírem rugas $(21,1 \%)$, cabelos grisalhos ou calvície $(20 \%)$ e por serem menos ativos $(17,5 \%)$. Estes estudantes possuíam uma visão mais positiva do seu próprio envelhecimento quando comparado com o de seus pais e outros idosos. É relevante notar que apenas $4,6 \%$ dos estudantes mencionaram doenças, $6 \%$ o uso de medicamentos e $5,7 \%$, problemas sensoriais.

Os achados da presente investigação sugerem que a dificuldade em oferecer cuidados ao idoso poderia estar relacionada às crenças e concepções dos trabalhadores em relação ao 'ser idoso'. Este aspecto deveria, portanto, ser investigado de forma eficiente no ato de contratação de funcionários para o trabalho com esta faixa etária. A despeito disso, os achados sugerem que a atitude do funcionário em relação ao idoso, ou seja, a maneira como ele o vê, exerce influência nas situações de cuidado. Estes resultados devem ser replicados em futuros estudos, para que se obtenha a consistência necessária à sua interpretação. 


\section{CONSIDERAÇÕES FINAIS}

As autoras deste estudo concordam com a autora ${ }^{(30)}$ que aponta sua indignação perante o cuidado prestado por cuidadores despreparados, o que desrespeita o idoso como pessoa e ainda impede qualquer tipo de reabilitação. Esta autora considera que a

implementação de programas inovadores para corrigir as distorções constatadas freqüentemente no cotidiano permitiriam aumentar a eficácia do atendimento domiciliar a partir do redimensionamento da rede assistencial existente, treinando inicialmente os prestadores de serviços nas instituições geriátricas e asilares que são cuidadores em potencial aos idosos no domicílio.

\section{REFERÊNCIAS}

1. Paschoal SMP. Autonomia e independência. In: Papaléo Netto M. Gerontologia. São Paulo: Atheneu; 1997. p. 313-23.

2. Neri AL. Atitudes em relação à velhice: evidências de pesquisas no Brasil. Gerontologia. 1997;5(3):130-9.

3. Incontri DA. A mídia e a imagem do idoso: uma questão de ética. In: Papaléo Netto M. Gerontologia. São Paulo: Atheneu; 1997. p. $458-62$.

4. Donato AF, Canôas SC. Idoso e cidadania: a lógica da exclusão. In: Papaléo Netto M. Gerontologia. São Paulo: Atheneu; 1997. p. 453-57.

5. Mercadante E. Aspectos antropológicos do envelhecimento. In: Papaléo Netto M. Gerontologia. São Paulo: Atheneu; 1997. p. 73-6.

6. Brito FC, Ramos LR. Serviços de atenção à saúde do idoso. In: Papaléo Netto M. Gerontologia. São Paulo: Atheneu; 1997. p. 394-402.

7. Born T, Boechat NS. A qualidade dos cuidados ao idoso institucionalizado. In: Freitas EV, Py L, Neri AL, Cançado FAX, Gorzoni ML, Rocha SM, editores. Tratado de geriatria e gerontologia. Rio de Janeiro: Guanabara Koogan; 2002. p. $768-77$.

8. Brasil. Ministério da Previdência e Assistência Social. Secretaria de Políticas de Assistência Social. Portaria n. 73, de 10 de maio de 2001. Define as normas de funcionamento de serviços de atenção ao idoso no Brasil. Diário Oficial da União, Brasília, 14 maio 2001. Seção 1, p. 174-84.

9. Born T. Cuidado ao idoso em instituição. In: Papaléo Netto M. Gerontologia. São Paulo: Atheneu; 1997. p. 403-14.

10. Yamamoto A, Diogo MJD'e. Os idosos e as instituições asilares do município de Campinas Rev Lat Am Enferm. 2002;10(5): 660-6.

11. Pavarini SCI. Dependência comportamental na velhice: uma análise do cuidado prestado ao idoso institucionalizado [tese]. Campinas: Faculdade de Educação, Universidade Estadual de Campinas; 1996.
Não há como negar que muitas das instituições de longa permanência estão passando por momentos críticos, incluindo a falta de infra-estrutura física e até o insuficiente número de pessoal de saúde qualificado. Não há como superar tantos problemas senão com a união do setor público e da sociedade civil, pois não há como exigir mudanças no sistema sem manutenção das instituições, engajamento com a causa do idoso, expresso em ações políticas, aplicação e liberação de recursos financeiros em programas de apoio à velhice e na luta para vencer as barreiras do preconceito ${ }^{(19)}$. Cabe ao profissional enfermeiro engajar-se ativamente nesse processo.

12. Diogo, MJD'E. Modalidades de assistência ao idoso e à família: impacto sobre a qualidade de vida. In: Freitas EV, Py L, Neri AL, Cançado FAX, Gorzoni ML, Rocha, SM, editores. Tratado de geriatria e gerontologia. Rio de Janeiro: Guanabara Koogan; 2002. p. 1065-8.

13. Pavarini SCI. Dependência comportamental na velhice: uma análise do cuidado prestado ao idoso institucionalizado [tese]. Campinas: Faculdade de Educação, Universidade Estadual de Campinas; 1996.

14. Duarte YAO. Pavarini SCI. Instituições de idosos: qualificação de pessoal. In: Anais do $1^{\circ}$ Congresso Paulista de Geriatria e Gerontologia; 1998 jun; São Paulo. p. 66-72.

15. Neri AL. Envelhecer num país de jovens: significado de velho e velhice segundo brasileiros não idosos. [tese livre-docência]. Campinas: Faculdade de Educação, Universidade Estadual de Campinas; 1988.

16. Duarte YAO. O idoso e o processo de envelhecimento sob a ótica do próprio idoso e de estudantes de enfermagem [dissertação]. São Paulo: Escola de Enfermagem, Universidade de São Paulo; 1996.

17. Fonseca RMGS, organizadora. Mulher e cidadania na nova ordem social. São Paulo: NEMGE/USP; 1996.

18. Duarte MJRS. Atenção ao idoso: um problema de saúde pública e de enfermagem. Rev Enferm UERJ. 1994;2(1):100-11.

19. Leão ALM, Adorno J, Vasconcelos LB. Instituições de apoio e assistência ao idoso em Goiânia: profissionais envolvidos e atuação do enfermeiro. Texto Contexto Enferm. 1997;6(2):352-8.

20. Kawasaki K, Diogo MJD'E. Assistência domiciliária ao idoso: perfil do cuidador formal - Parte I. Rev Esc Enferm USP. 2001;35(3):257-64.

21. Franqueiro NVJ. Formação gerontológica em um curso de graduação em enfermagem: análise curricular mediante as novas diretrizes da educação [dissertação]. Ribeirão Preto: Escola de Enfermagem de Ribeirão Preto, Universidade de São Paulo; 2002. 
22. Freitas MC. Processo de formação do enfermeiro e o envelhecimento humano: conhecimento e interesses de educadores e educandos [tese]. Ribeirão Preto: Escola de Enfermagem de Ribeirão Preto, Universidade de São Paulo; 2003.

23. Macedo CP. Prática dos enfermeiros na assistência ao idoso, nas unidades básicas de saúde de Belo Horizonte [dissertação]. Belo Horizonte: Escola de Enfermagem, Universidade Federal de Minas Gerais; 2003.

24. Associação Brasileira de Enfermagem (ABEn). A nova lei do exercício profissional da enfermagem: uma análise crítica. Brasília: ABEn; 1987. p. 20-8.

25. Pavarini SCI, Neri AL. Compreendendo dependência, independência e autonomia no contexto domiciliar: conceitos, atitudes e comportamentos. In: Duarte YAO, Diogo MJD'E. Atendimento domiciliar: um enfoque gerontológico. São Paulo: Atheneu; 2000. p. 49-70.
26. Berger L. Atitudes, mitos e estereótipos In: Berger L, MailouxPoirer D. Pessoas idosas: uma abordagem global. Lisboa: Lusodidacta; 1995. p. 63-71.

27. Ebersole P, Hess P. Toward healthy aging. $5^{\text {th }}$ ed. St. Louis: Mosby; 1998.

28. Baltes MM, Neumann EM, Zank S. Maintenance and rehabilitation of independence in old age: an intervention program for staff. Psychol Aging. 1994;9(2):179-88.

29. Lichtenstein MJ, Pruski LA, Marshall CE, Blalock CL, Lee S, Plaetke R. Sentence completion to assess children's views about aging. Gerontologist. 2003;43(6):839-48.

30. Duarte MJRS. Cuidadores? Por que e para quê? Atenção ao idoso no domicílio. Rev Enferm UERJ. 1996;4(n. esp.):126-30.

Agradecimentos: à Profa. Dra. Maria José D'Elboux Diogo, pela colaboração essencial ao desenvolvimento desta pesquisa; à FAPESP, pelo apoio financeiro. 